\title{
Characterizing the velocity field in hydrodynamical simulations of low-mass star formation using spectral line profiles
}

\author{
C. Brinch ${ }^{1}$, M. R. Hogerheijde ${ }^{1}$, and S. Richling ${ }^{2}$ \\ ${ }^{1}$ Leiden Observatory, Leiden University, PO Box 9513, 2300 RA Leiden, The Netherlands \\ e-mail: brinch@strw. leidenuniv.nl \\ 2 Institut d'Astrophysique de Paris, 98 bis Boulevard Arago, 75014 Paris, France
}

Received 23 April 2008 / Accepted 10 June 2008

\section{ABSTRACT}

\begin{abstract}
Context. When low-mass stars form, the collapsing cloud of gas and dust goes through several stages which are usually characterized by the shape of their spectral energy distributions. Such classification is based on the cloud morphology only and does not address the dynamical state of the object.

Aims. In this paper we investigate the initial cloud collapse and subsequent disk formation through the dynamical behavior as reflected in the sub-millimeter spectral emission line profiles. If a young stellar object is to be characterized by its dynamical structure it is important to know how accurately information about the velocity field can be extracted and which observables provide the best description of the kinematics. Of particular interest is the transition from infalling envelope to rotating disk, because this provides the initial conditions for the protoplanetary disk, such as mass and size.

Methods. We use a hydrodynamical model, describing the collapse of a core and formation of a disk, to produce synthetic observables which we compare to calculated line profiles of a simple parameterized model. Because we know the velocity field from the hydrodynamical simulation we can determine in a quantitative way how well our best-fit parameterized velocity field reproduces the original. We use a molecular line excitation and radiation transfer code to produce spectra of both our hydrodynamical simulation as well as our parameterized model.

Results. We find that information about the velocity field can reasonably well be derived by fitting a simple model to either singledish (15" resolution) lines or interferometric data (1" resolution), but preferentially by using a combination of the two. The method does not rely on a specific set of tracers, but we show that some tracers work better than others. Our result shows that it is possible to establish relative ages of a sample of young stellar objects using this method, independently of the details of the hydrodynamical model.
\end{abstract}

Key words. radiative transfer - hydrodynamics - stars: formation - ISM: molecules - line: profiles

\section{Introduction}

Low-mass young stellar objects (YSOs) are well-studied phenomena. Observationally, these objects have been measured in many different wavelength regimes in order to determine intrinsic properties such as their mass, luminosity, mass accretion rate, etc. YSOs are traditionally classified by the shape of their spectral energy distribution (SED) (Lada \& Wilking 1984), which reflects their general morphology. Subsequently, the envelope and disk densities, temperatures and chemistry can be studied using millimeter continuum and molecular line measurements. All of these derived quantities have been used to piece together a consistent chronology of the process of star formation and to establish a reference from which the age of a YSO can be reliably obtained (Mardones et al. 1997; Gregersen \& Evans 2000).

A theoretical evolution scenario of YSOs has been established for many years (e.g., Ulrich 1976; Shu 1977; Cassen \& Moosman 1981; Terebey et al. 1984; Bodenheimer et al. 1990; Basu 1998; Yorke \& Bodenheimer 1999). In these models (with the exception of the Shu model, which is spherically symmetric) a circumstellar disk is formed, after the collapse of the initial cloud sets in, due to conservation of angular momentum. It is therefore natural to use the kinematic properties of the models to characterize the evolutionary stage of the object, because the distribution of angular momentum changes in a monotonous way as the cloud contracts and matter spins up. Unfortunately, measuring the velocity field observationally is not such a simple matter. Kinematic information can only be derived from spectral emission lines and there is no way to directly solve for the velocity field from measurements of such lines. The reason for this is that the spectral profile depends on a range of physical parameters, including the local density, temperature, molecular abundance, and turbulence in the region where the transition in consideration is excited. All of this can be taken into account and self-consistent models, including a general parameterized velocity field, can be built and fitted to the observed spectra. The question is, however, how reliable the derived velocity field is given the input model, and to what extent the derived velocity fields are consistent with the prediction of the models.

The velocity field of YSOs has been well studied observationally in the last 20 years. At first, mainly infall was observed (e.g., Calvet \& Hartmann 1992; van Langevelde et al. 1994), but soon afterward, objects showing a mix of infall and rotation was discovered (Saito et al. 1996; Ohashi et al. 1997; Hogerheijde 2001; Belloche et al. 2002). More recently, protoplanetary disks have been studied in very high resolution using sub-millimeter interferometry and many of these have velocity fields with rotation only (e.g., Lommen et al. 2008). The common interpretation of these observations is that the objects showing infall only are early-type embedded objects, the rotating disks are the end 
product of the collapse, and the objects showing both infall and rotation are in some kind of transition. Initial modeling work using a parameterization for the velocity field has been done by Myers et al. (1996) for low-mass star formation and by Keto (1990) for the formation of high-mass stars. However, it has never been attempted to quantify the degree of the transition, how far the object has passed from the embedded phase to the protoplanetary disk phase.

We are addressing this question by comparing synthetic spectra, calculated from a hydrodynamical simulation of a collapsing cloud, to a model with a simple parameterized velocity field. The comparison is done in spectral space in the same way as one would compare a model to real observations. By considering different transitions of different molecules in two different resolution settings, we investigate how to obtain the most reliable characterization of the velocity field. The importance of being able to reliably model the observations of YSOs using a simple generic model becomes evident when considering the capabilities of the upcoming ALMA (Atacama Large Millimeter Array). While it is possible to build sophisticated custom models, or even directly use the hydrodynamical simulation as a template model to describe single objects, this is a very timeconsuming process. ALMA will produce very high resolution observations $\left(\sim 0.01^{\prime \prime}\right)$ in snapshot mode and in order to deal with such vast samples of YSOs, simple yet reliable models are needed.

The layout of this paper is as follows: in Sect. 2 we present the hydrodynamical simulation of Yorke \& Bodenheimer (1999) that we use to make synthetic observations and describes our model and the way we fit this to the synthetic spectra. Section 3 presents the synthetic observables and also a few analytical examples of idealized cases for comparison. Section 4 shows our results of the spectral comparison and the derived velocity fields, followed by a discussion and conclusion in Sects. 5 and 6, respectively.

\section{Simulations and models}

\subsection{Hydrodynamical collapse}

For the description of the gravitational collapse, we use the hydrodynamical scheme of Yorke \& Bodenheimer (1999). In particular we use the model which simulates the formation of an J-type star given an initial cloud mass of $1 M_{\odot}$ and radius of $6667 \mathrm{AU}\left(1 \times 10^{15} \mathrm{~m}\right)$. The cloud is initially isothermal at $10 \mathrm{~K}$ and is given a constant solid body rotation of $1 \times 10^{-13} \mathrm{~s}^{-1}$. In this model, an equilibrium disk is formed after about 75000 years after the onset of the collapse, which then grows up to several thousands of AU as angular momentum is transported outwards. The disk which is formed resembles a thin, flaring disk with a pressure scale height at $500 \mathrm{AU}$ from the star of $h=0.15 R$ and $h=0.19 R$ at $1000 \mathrm{AU}$ from the star.

The code used to calculate the collapse is described in detail by Różyczka (1985), Yorke et al. (1993, 1995), and Yorke \& Bodenheimer (1999), but a brief overview is provided here as well. The standard equations of hydrodynamics including radiative transfer and the Poisson equation for the gravitational potential is solved on five regular nested grids of 68 by 68 cells. The grid does not resolve the central star, but the centermost grid cell is treated as a sink into which mass and angular momentum can flow. The stellar luminosity is calculated from the mass and mass accretion of this cell and the luminosity is used as a boundary condition for the radiative transfer calculations. The radiative transfer is solved using the frequency dependent flux-limited diffusion method (for details, see Yorke \& Bodenheimer 1999), in order to obtain a self-consistent temperature distribution. Artificial viscosity is included in order to deal with shocked regions and physical viscosity is provided by an $\alpha$ prescription (Shakura \& Sunyaev 1973) to include angular momentum transport in the disk.

We use the initial free fall time scale to describe the age of the simulated YSO. Free fall time is given as

$t_{\mathrm{ff}}=\sqrt{\frac{\pi^{2} R^{3}}{8 G M}}$,

which for this particular model is approximately $1 \times 10^{5}$ years. The simulation can roughly be divided into three parts, namely the infall dominated pre-disk stage at $0.5 t_{\mathrm{ff}}$, the disk formation stage at about $1.0 t_{\mathrm{ff}}$ in which both infall and rotation are important, and the rotation dominated disk growth stage at $2.0 t_{\mathrm{ff}}$. The entire simulation runs for about $2.5 t_{\mathrm{ff}}$ at which point it halts. Representative snapshots of the density, temperature, and velocity structure at these three stages are shown in Fig. 1. In the three panels, the temperature and density contours are kept at the same values for comparison.

We have selected a number of snapshots throughout the simulation, 13 in total evenly spaced by about 0.2 in free-fall time, as input for our molecular excitation and frequency dependent radiation transfer code RATRAN (Hogerheijde \& van der Tak 2000). This code runs on a similar, but coarser grid, so the density, temperature and velocities of the hydrodynamical simulation can directly be remapped onto a RATRAN grid. The level population in each cell is then solved iteratively by propagating photons randomly through the grid until the level populations are in equilibrium with the radiation field.

The reason why we do not use every snapshot (several hundreds, depending on the time resolution in the output) is that the molecular excitation calculations are quite time consuming. The CPU time required to determine the level populations for a single snapshot is comparable to the CPU time it takes to run the entire hydrodynamical simulation, which is the reason why such accurate radiation transfer methods are not included in the simulation itself. However, the level populations and hence the line profiles do not change dramatically from one time step to the next, so there is actually no need, for our purpose, to use more than one snapshot per $0.1-0.2 t_{\mathrm{ff}}$.

In RATRAN, a molecular abundance is added to the model reflecting the chemical abundance of the molecule in question. The simplest possible abundance description uses a constant average abundance relative to the $\mathrm{H}_{2}$ density which is how we populate the models with molecules in this paper. More realistic abundance profiles, based on temperature and density dependent freeze-out and desorption rates, have been discussed by, e.g., Lee et al. (2004) and Jørgensen et al. (2004). In Sect. 5 we discuss the effects on our results, if a more realistic abundance distribution is added to the synthetic observations. For an in-depth treatment of the inclusion of depletion chemistry in the hydrodynamical simulation and its effect on the resulting spectra, we refer the reader to the subsequent paper by Brinch et al. (2008).

We execute separate RATRAN calculations for different molecular species. As mentioned above, these calculations are relatively time consuming, so we have chosen to focus on only three commonly observed molecules, $\mathrm{CO}, \mathrm{HCO}^{+}$, and $\mathrm{CS}$, and their isotopologues. These three species probe different densities and have different optical depth and therefore the spectral profiles come out very different even for the same underlying snapshot. We can of course repeat the entire exercise for any 

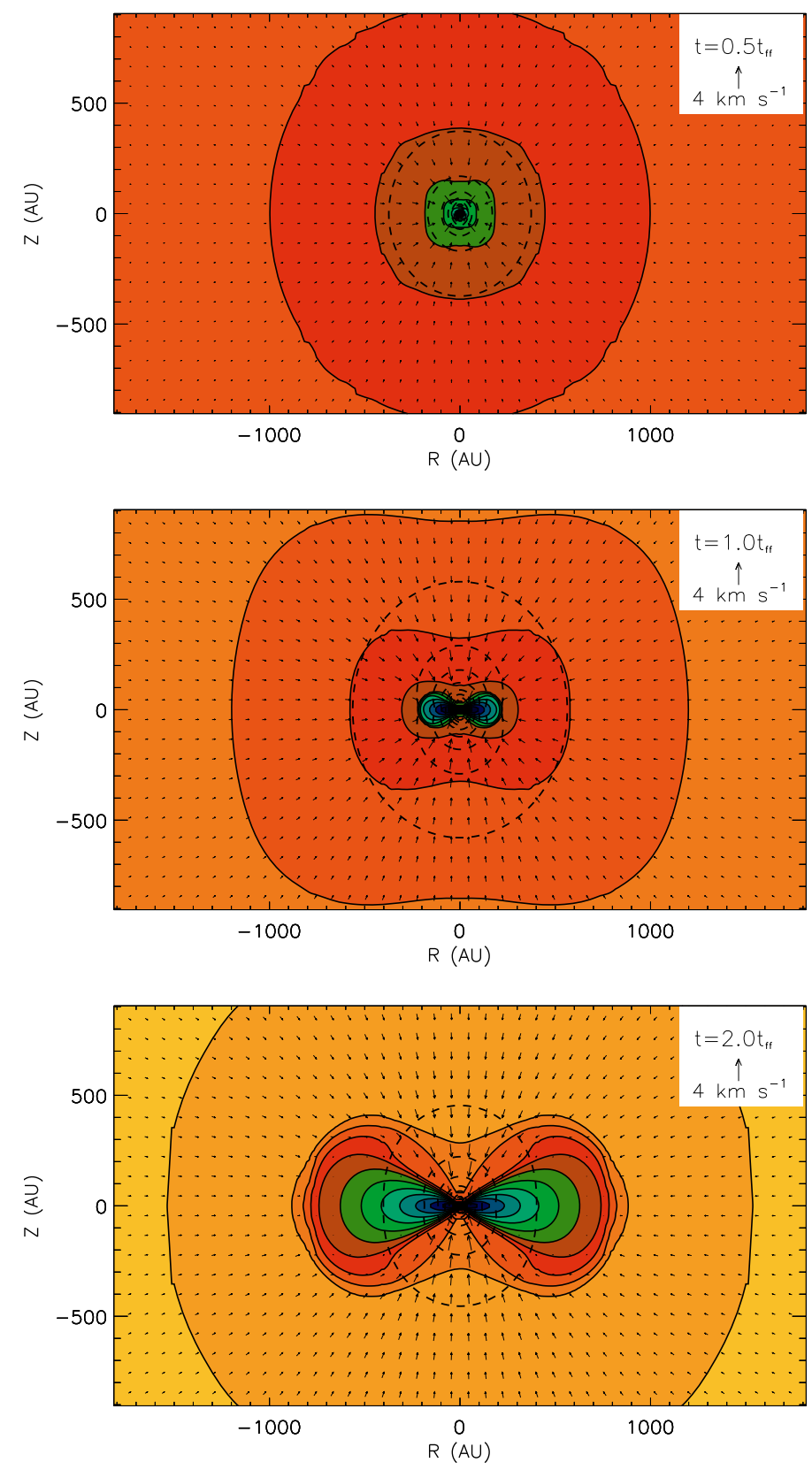

Fig. 1. Model structure at three different ages. Full contours are the density starting at $3 \times 10^{5} \mathrm{~cm}^{-3}$ and increasing with $\log \rho=0.5$. The temperature is shown as dashed contours, starting at $20 \mathrm{~K}$ and increasing with $10 \mathrm{~K}$. The arrows show the infall component of the velocity field.

molecule for which collisional rates are available and this makes our method very flexible. The collisional rates used in this paper come from the LAMDA database ${ }^{1}$ (Schöier et al. 2005). When the level populations have converged, the model is ray-traced in frequency bands around the desired transitions to obtain spectral image cubes from which spectral lines can be extracted. In the following we consider increasingly higher transitions for the three species, so that these not only probe increasingly higher densities but also increasingly higher temperatures.

We do not claim that this hydrodynamical collapse model provides a particularly realistic description of nature. Neither do we try to fit this model to observation, although in principle, the method presented in this paper can be used to compare and

1 Available at http://www . strw. leidenuniv.nl/ moldata constrain hydrodynamical models with molecular line observations. Instead, we employ the simulation to provide a time resolved velocity field topology going from an infall dominated field to a rotation dominated field. The scope of this paper is to show how the velocity field is reflected in the emission line profiles and to see to what extent the velocity field can be reconstructed from these lines. As long as the hydrodynamical simulation approximates reality, our method is valid. The two main caveats of our simulation are the lack of any kind of mass loss through either a wind or an outflow and the unknown initial condition for the angular momentum distribution. The lack of mass loss means that all the mass that is initially in the model ends up in either the disk or the star, and especially the disk ends up being very large and massive. An outflow would not only carry away mass but also angular momentum and the result of this would be a smaller, less massive disk. The effect of the second item, the initial angular momentum distribution is somewhat more uncertain and a point of further study, which however is beyond the scope of this paper.

\subsection{Parameterized model}

We have chosen a particularly simple parameterization for the model which we use to derive the velocity field. This model is a simplified version of the one used by Brinch et al. (2007) to interpret single dish observations of the YSO L1489 IRS.

In this paper we use a spherical version of this model, where both the density and the temperature are given by power-laws

$$
\begin{aligned}
n & =n_{0}\left(r / r_{0}\right)^{-p}, \\
T & =T_{0}\left(r / r_{0}\right)^{-q},
\end{aligned}
$$

which means that our parameterized model neglects the flattening of the disk. We assume that the inclination is known to at least $10-20 \%$. Although our model is spherically symmetric, it still has a rotation axis, which may be inclined with respect to our line of sight. For the remainder of this paper we adopt an edge-on view of the simulation $\left(i=90^{\circ}\right)$. The velocity field is described by two free parameters, the central mass $M_{*}$ and an angle $\alpha$, that is defined as,

$\alpha=\arctan \left(\frac{v_{r}}{v_{\phi}}\right)$,

where $v_{r}$ and $v_{\phi}$ are the free-fall and Keplerian velocity components, respectively, given by

$v_{\phi} \cos (\alpha)=-\frac{v_{r}}{\sqrt{2}} \sin (\alpha)=\sqrt{\frac{G M_{*}}{r}}$.

The angle $\alpha$ is thus the angle of the resulting velocity field vectors with respect to the azimuthal direction, and therefore the ratio of infall to rotation. In other words, if $\alpha=0$ the cloud is in pure Keplerian motion whereas if $\alpha=\pi / 2$ the cloud is collapsing in free fall. This parameterization is allowed because both rotation and infall have a $\sqrt{M_{*} / r}$ dependency.

The advantage of this simple parameterization is that it has few free parameters and, more importantly, for a large part of the hydrodynamical simulation the density and temperature are actually very well described by power-laws. The disadvantage is of course that it cannot describe the disk structure at all. However, since we are aiming at characterizing the velocity field, the exact shape and distribution of disk material is less important and it turns out that is has little impact on our result as long as we fit 
our density model to the mid-plane density of the hydrodynamical output.

The $\alpha$ parameter has no direct equivalent in the hydrodynamical simulation because the ratio of infall to rotation is not generally constant with radius. In the remainder of this paper we do however use an averaged value for $\alpha$ where we have radially averaged the individual $\alpha$ values along the mid-plane in order to evaluate the quality of our results.

In this paper we assume that the inclination is known (we consider only the edge-on case, but our result holds true except for inclinations very close to face-on). In the beginning of the simulation, where rotation is less important, the inclination is harder to constrain observationally, but on the other hand, it does not really matter since the object is mostly spherical anyway. As soon as a disk has formed, the inclination can easily be constrained by modeling the SED or from near-infrared imaging. In any case, if the inclination is not known, it can in principle be included as a free parameter at the cost of computation time.

A final caveat of this simple model is the flat abundance distribution. As discussed above, the abundance distribution will evolve dynamically with the evolution of the temperature and density, and this will have an effect on the spectral lines. This effect can be taken into account in the modeling using the approach of Jørgensen et al. (2004), but for the sake of clarity we will disregard the chemistry in the main part of this paper.

\subsection{Obtaining the best fit}

When fitting the parameterized model to the hydrodynamical solution, we do so in a sequence of comparisons between different synthetic observables. First we constrain the five parameters $n_{0}, T_{0}, p, q$, and $R_{\text {out }}\left(R_{\text {out }}\right.$ is the outer radius of the model beyond which the power-laws are truncated) in the density and temperature model by fitting radial continuum profiles at 450 and $850 \mu \mathrm{m}$. A synthetic continuum image is calculated by RATRAN at each wavelength based on the hydrodynamical solution. Similarly we calculate images for the parameterized model that is then evaluated with respect to the image from the hydrodynamical solution by calculating the $\chi^{2}$ measure. In other words, we obtain the set of parameters which gives the best representation of the hydrodynamical structure in the image space. The wavelengths and image resolution are chosen so that the images mimic real observations by instruments such as SCUBA on the James Clerk Maxwell Telescope (15 m single dish, with a typical resolution of $15^{\prime \prime}$, corresponding to $2000 \mathrm{AU}$ at the distance of the Taurus star forming region).

When the physical structure has been determined we fix the molecular abundance by fitting an emission line of an optically thin isotope. In the case of $\mathrm{HCO}^{+}$, this isotope would for example be $\mathrm{H}^{13} \mathrm{CO}^{+}$, whereas for $\mathrm{CO}$ we could use $\mathrm{C}^{17} \mathrm{O}$. An optically thin line traces the total column density and can as such be used to derive the average abundance. Since we have not yet determined the velocity field, we aim at fitting the integrated intensity rather than the peak intensity or the line profile itself. The procedure is the same as before: a single spectrum towards the center is calculated by RATRAN based on the hydrodynamical solution which is then used as a synthetic observation to fit the same spectrum based on the parameterized model. Again, we use a spectral and spatial resolution which is typical for existing single dish telescopes $\left(15^{\prime \prime}\right)$.

Finally, when the abundance is fixed, we fit the velocity field parameters. This is done by comparing optically thick lines. In the case where we simulate single dish observations, we use only a single spectrum towards the center of the object, in which case we fit only the central mass and a single angle $\alpha$. If we use simulated interferometric observations, where there are typically several resolution elements across the object, we fit spectra which are separated by one synthesized beam, with as many corresponding angles $\alpha$.

In order to fully explore the parameter space to reach the optimal solution, we use the genetic algorithm PIKAIA (Charbonneau 1995) which is a stochastic optimizer. This algorithm is applied in each step described above and is very successful in finding the true minimum of the search space. The benefit of using this algorithm for this purpose is that it takes a relative low number of model calculation to reach the optimal solution compared to the more traditional "brute force" method where a grid of models are calculated. Since calculating a model spectrum takes of the order minutes to complete the applicability of our method is highly dependent of the number of models which are required to be calculated. Another benefit of using a code such as PIKAIA is that the entire parameter space gets well sampled which enables us to determine the $\chi^{2}$ distribution along each parameter axis. The resulting distributions are approximated by Gaussians, and the $F W H M$ of these are used to get a handle on the robustness of the fit. It should be noted that this is not an error bar in the sense that our model fit is consistent with the synthetic data if it falls within the range of the $F W H M$ of the Gaussian approximation to the $\chi^{2}$ distribution. These numbers only show how sharply defined the minimum of the parameter space is for a specific optimization.

Figure 2 shows how well we are able to approximate the hydrodynamical solution by our simple parameterized model. The example shown is just a randomly chosen snapshot $\left(t=1.0 t_{\mathrm{ff}}\right)$.

\section{Line profiles and the velocity field}

Initially the cloud collapse model is given a solid body rotation. As gravitation sets in and infall velocity builds up towards free fall, matter is redistributed and so is the angular momentum. Material ends up in the plane of rotation, where it spins up, approaching the Keplerian velocity. At this point, the matter cannot be given a higher angular momentum, so as matter continuously moves inward from outside of the disk, angular momentum is transported outwards through the disk resulting in growth of the disk.

In Fig. 3 the radial and the azimuthal components of the velocity field in the disk mid-plane at a radius of 500 AU are plotted. Also shown in this plot is the free-fall and Keplerian velocities calculated as a function of the central core mass which grows in time as mass is being accreted onto the star. Initially, the radial velocity builds up faster than the azimuthal velocity component, but at an age of $t=1.5 t_{\mathrm{ff}}$ the disk expansion shock wave passes $500 \mathrm{AU}$ and afterward the region is completely dominated by rotational velocity close to the Keplerian. Indeed the velocity is seen to be slightly super-Keplerian after the expansion wave passes because of the outwards angular momentum transport. Similarly, Fig. 4 shows again the infall and rotation velocity profiles, but for a single time slice $\left(t=1.0 t_{\mathrm{ff}}\right)$. In this figure, the velocities are plotted against the mid-plane radius and also shown are the free-fall and Keplarian profiles, indicated by thin lines. The disk radius is clearly seen by the break in the infall profile around $200 \mathrm{AU}$. It is also seen that the velocity field is very well approximated by free-fall outside of the disk radius, whereas the inside of the disk radius is well described by Keplerian motion.

The velocity of a gas is reflected in the shape of the emission line profiles. This includes both the random particle motion and the systematic motion due to potential gradients. For the 

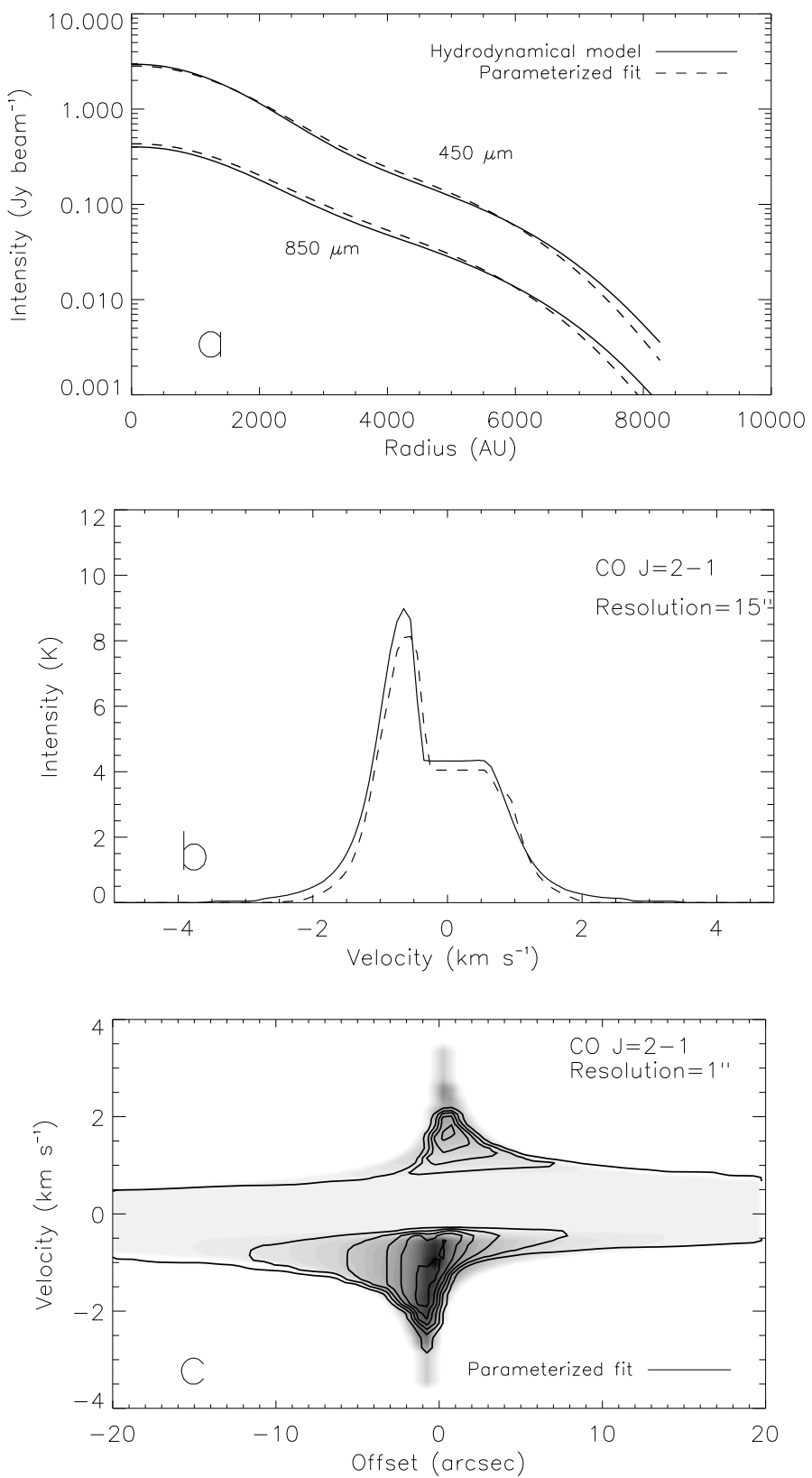

Fig. 2. Example of a fit by our parameterized model to the hydrodynamical solution at $t=1.0 t_{\mathrm{ff}}$ for the case of $\mathrm{CO}$. The full lines are the output of the hydrodynamical simulation and the dashed lines are the fit by our parameterized model. In panel c), the hydrodynamical output is shown by the gray scale and the model fit by contour lines.

model cloud considered here, the former is of minor importance although we do include a microturbulent field with a Gaussian $F W H M$ of $0.2 \mathrm{~km} \mathrm{~s}^{-1}$ in our calculations. The systematic motion is of course our main interest here, because this is what governs the dynamics of the cloud.

Initially, the cloud is collapsing in a nearly spherical symmetric way which is entirely dominated by radial motions. This should be reflected in the line profiles as the characteristic infall asymmetry. In the case of optically thick lines, the difference in temperature of the red shifted and the blue shifted peaks provides a measure of the optical depths (e.g., Evans 1999). In the latest stage, when most of the gas has been accreted onto the disk which is dominated by rotation, the line profiles are expected to be double peaked, with both peaks having the same intensity. At

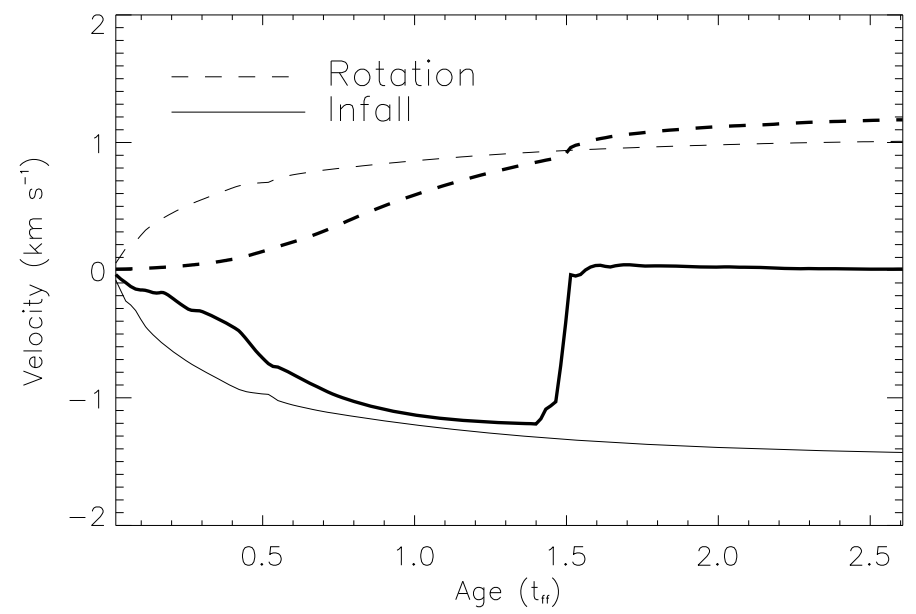

Fig. 3. The rotation and infall velocities of the collapse simulation in the mid-plane at a radius of $500 \mathrm{AU}$ plotted as a function of free-fall time. The velocities of the simulation are shown in thick lines. In this figure we also show the Keplerian and free-fall velocities which are both functions of the central mass. These are shown as thin lines.

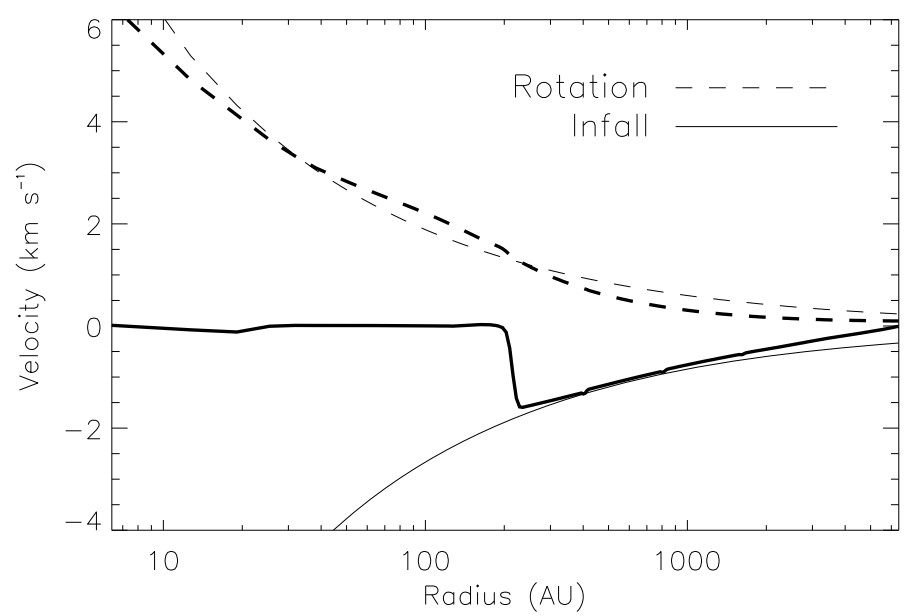

Fig. 4. The rotation and infall velocities in the simulation along the midplane at an age of $t=1.0 t_{\mathrm{ff}}$. As in Fig. 3, the thin lines correspond to free-fall and Keplerian velocity profiles.

this point, gas has reached such a low density outside of the disk, that we do not expect to see any contribution to the line from radial motions. In the intermediate stage however, where the disk is still accreting significantly, both velocity components, radial and azimuthal, will contribute to the spectral line profile. The exact shape of such a profile is hard to predict because it is critically dependent on the exact optical depth, the critical density of the transition, the spatial resolution, and the system inclination. This is further complicated by the fact that no analytical model exists of the transition from a collapsing envelope to a viscously supported protoplanetary disk.

As an illustration, we show model spectra in Fig. 5 which have been calculated with a model which is either in free fall (with no azimuthal velocity component at all) or in Keplerian rotation (no radial components). The top-most panels in this figure show the infalling models in which the infall-asymmetry (Evans 1999 ) is clearly seen. The corresponding position-velocity diagram also resembles the CO 2-1 at $t=0.5 t_{\text {ff }}$ panel in Fig. 8 . Similarly, the PV-diagram in the lower row of Fig. 5 resembles the late stage PV-diagram in Fig. 8 and the low-resolution spectrum shows the double-peak feature which is associated with 

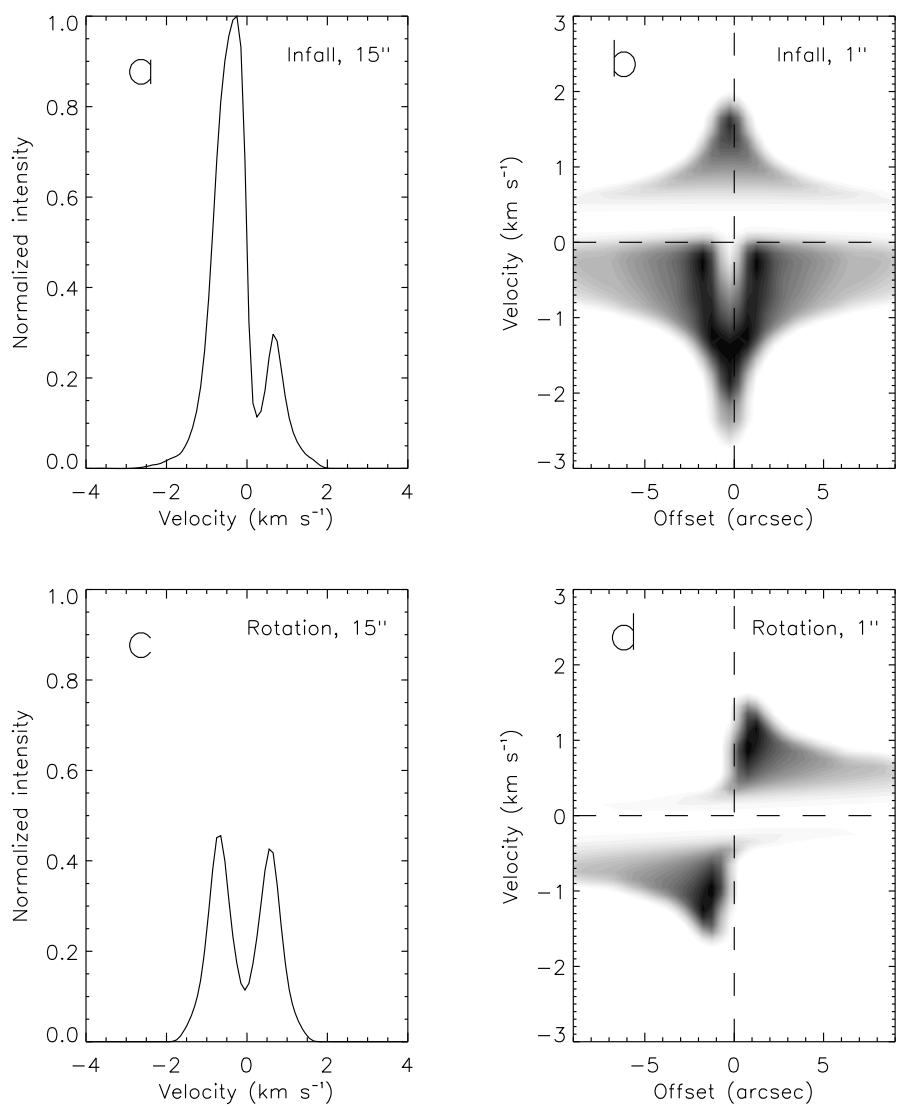

Fig. 5. Low-resolution spectra and high-resolution position-velocity diagrams of a purely (free) infalling model and a purely (Keplerian) rotating model.

rotation. Therefore, if the gas has the particular density in which an observed transition is strongly dominated by one or the other type of motion, it is possible to tell the difference from the shape of the line or the intensity distribution of the PV-diagram. This, however is rarely the case because the velocity field in general changes with increasing density (due to angular momentum conservation) and in most cases, the gas has non-vanishing velocity components in all three spatial directions.

\subsection{Low resolution spectra}

Using our hydrodynamical simulation as input model we can calculate spectra of any disk and envelope configuration ranging from purely collapsing to entirely rotating. In Fig. 6 is shown the result of such a calculation. These spectra are convolved with a $15^{\prime \prime}$ FWHM Gaussian beam so that they are comparable to observed spectra obtained with a typical large single dish telescope. The system inclination has been chosen to be exactly edge-on so that the viewing angle is the same as in Fig. 1.

In the leftmost panel of Fig. 6 are shown CO $J=2-1$ spectra based on the three time snapshots from Fig. 1. The CO lines are seen to be very optically thick throughout the simulation which is a result of the relatively high $\mathrm{CO}$ abundance. Actually, the optical depth of the CO lines is so high that we do not even see the rotating disk in the last snapshot, where the profile still bears the typical infall signature also seen in the two earlier snapshots. Even though the densities are very low $\left(<10^{4} \mathrm{~cm}^{-3}\right)$ outside of the disk at $t=2.0 t_{\mathrm{ff}}$, there is still enough column to entirely mask the disk. In the middle panel, $\mathrm{HCO}^{+} J=3-2$ spectra are shown. These are optically thick too, but not as much as
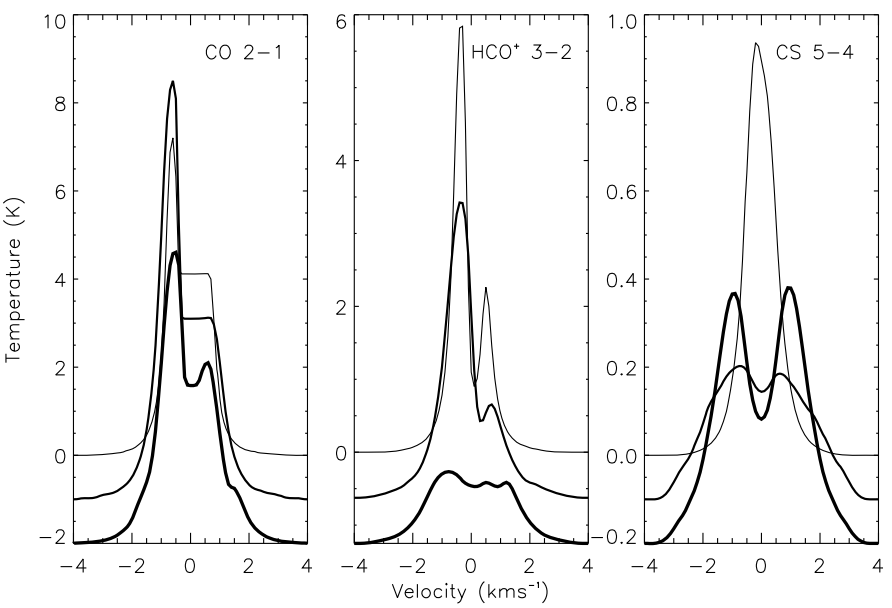

Fig. 6. Spectral line profiles of three different tracers at three different ages. $t_{\mathrm{ff}}=1.0$ and 2.0 are offset by $-10 \%$ and $-20 \%$ respectively. These spectra have been convolved with a 15" Gaussian beam to resemble typical single-dish telescope observations.

the CO lines. The infall asymmetry is very pronounced in the $t=0.5 t_{\mathrm{ff}}$ spectrum and is still visible in the $t=1.0 t_{\mathrm{ff}}$. In the last snapshot, the infall signature is gone, and we only see a flattened Gaussian-like spectrum. In the right-most panel of Fig. 6 we have plotted CS $J=5-4$. This line traces very high densities $\left(>10^{6} \mathrm{~cm}^{-3}\right)$. Notice that the intensity of these lines are about an order of magnitude lower than the intensity of the CO lines. In the earliest snapshot, this transition is actually optically thin and only a hint of asymmetry is seen here. As we go to later stages, the CS lines also start to become optically thick. This line, however, clearly displays a double peaked profile in the late stage. We also see, contrary to the $\mathrm{CO}$ and $\mathrm{HCO}^{+}$lines that the width of the CS spectra increases with time.

To illustrate how the shape of the line profile is a reflection of the underlying velocity field, we have quantified the line asymmetry by measuring the intensity difference between the two peaks, normalized with the peak intensity of the line. This gives us a dimensionless number between 0 and 1 , where 0 corresponds to the extreme asymmetric line and 1 is a perfectly symmetric line. We have plotted this quantity for the whole time series of model spectra of $\mathrm{CO}, \mathrm{HCO}^{+}$, and $\mathrm{CS}$ in Fig. 7. These line asymmetries are plotted as dotted lines. The full line in Fig. 7 is the radially averaged angle $\alpha$ from the hydrodynamical simulation as calculated by Eq. (4). The Pearson correlation coefficients between $\alpha$ and line profile asymmetry are $22 \%, 61 \%$, and $83 \%$ for $\mathrm{CO}, \mathrm{HCO}^{+}$, and $\mathrm{CS}$, respectively, meaning that the asymmetry of the CS lines is a quite well correlated with the ratio of infall to rotation. Similarly, the central stellar mass (as given by the hydrodynamical simulation) can be correlated with the FWHM of the lines, resulting in correlation coefficients of $96 \%, 63 \%$, and $89 \%$ respectively; also a very high degree of correlation.

\subsection{High resolution spectra}

With the use of present day and future sub-millimeter interferometers, it is possible to obtain spatially resolved observations of protostellar cores and protoplanetary disks. Using this kind of instruments, resolutions of $1^{\prime \prime}$ or better are possible. When a resolved spectral image cube is available, it is possible to plot the velocity off-set as a function of projected radius by contouring the measured intensity in position-velocity space. Figure 8 shows PV-diagrams of three time snapshots of each of the three 


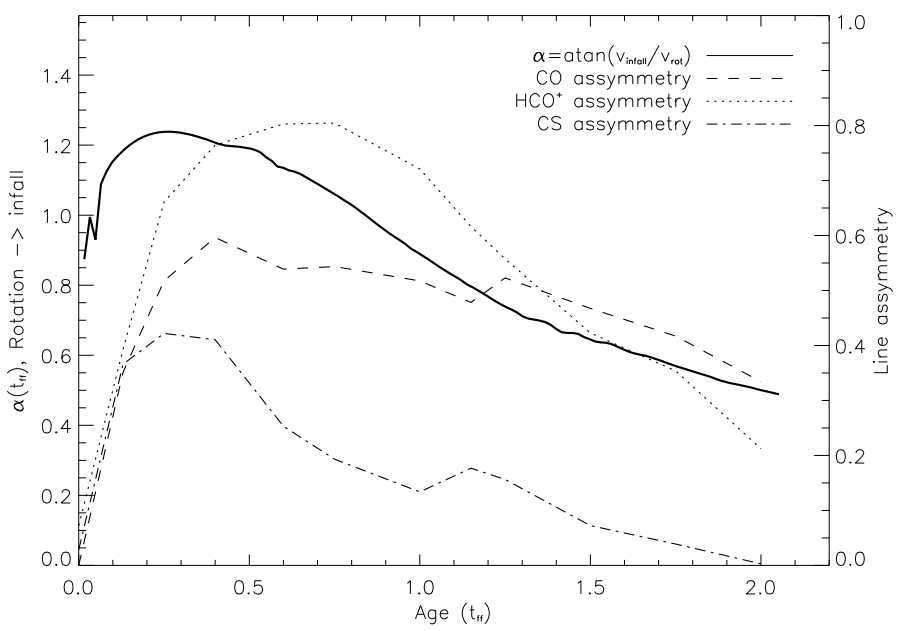

Fig. 7. The averaged $\alpha$ from the hydrodynamical simulation plotted against free-fall time (thick solid line). The punctured lines show the dimensionless asymmetry of the single-dish spectral lines as a function of time.

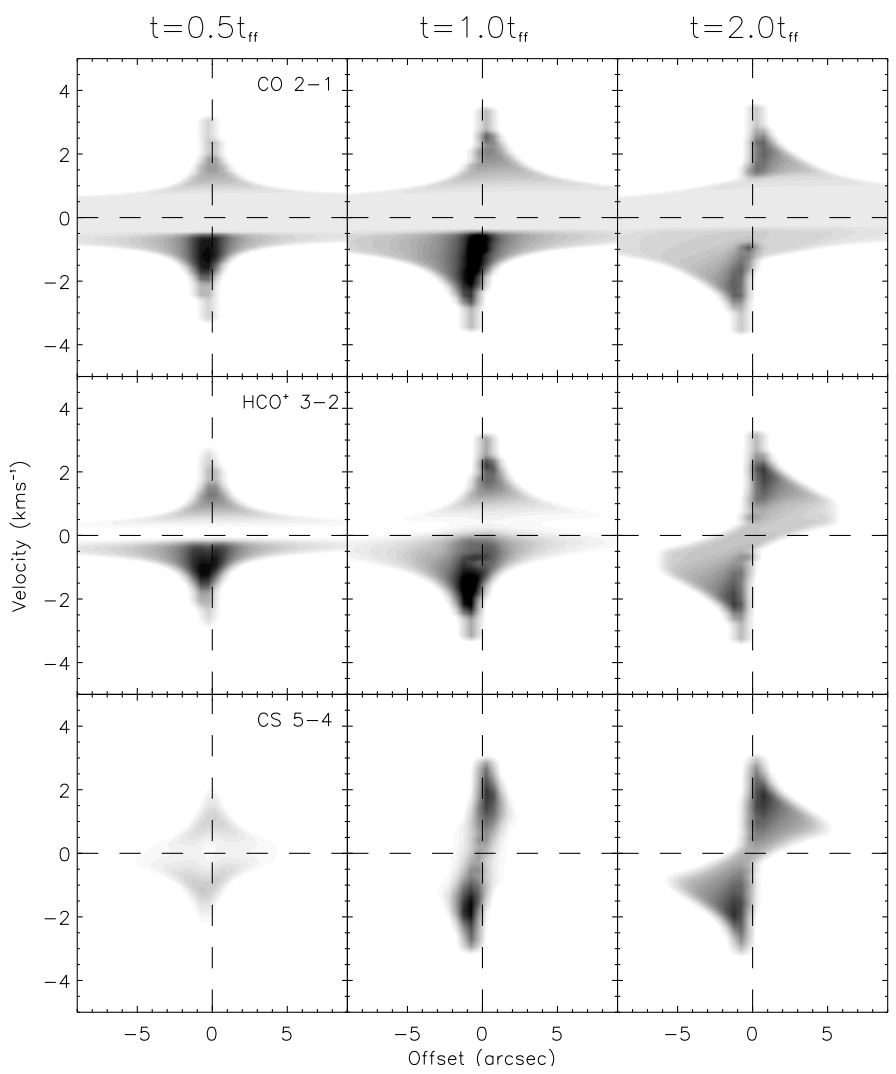

Fig. 8. High resolution position-velocity diagrams of CO 2-1, $\mathrm{HCO}^{+} 3-2$, and CS 5-4 at three different stages of the collapse simulation. These spectra have been convolved with a 1" FWHM Gaussian beam so that the resolution, in these diagrams, are comparable to the typical sub-millimeter interferometer resolution.

transitions: $\mathrm{CO} 2-1, \mathrm{HCO}^{+} 3-2$, and $\mathrm{CS} 5-4$. As in Fig. 6 we see a clear evolution in these diagrams, both in time and with increasing excitation energy. In the earliest snapshot, there is emission in almost equal amounts in all four quadrants, whereas in the latest snapshot, and most pronounced in the CS transition, the emission contours are butterfly-shaped and confined to the first and third quadrants. The former is typical for radial motion only while the latter is associated with rotation.
Although it is to some extent possible to characterize the velocity field that results in a particular PV-diagram, it is clear from the $t=1.0 t_{\text {ff }}$ panels in Fig. 8 that the answer one gets depends on the molecule in consideration. At $t=1.0 t_{\mathrm{ff}}$ one would conclude from the $\mathrm{CO}$ transition that the cloud is contracting whereas from CS the conclusion would be more or less pure rotation. The reason for this is that these two transitions are excited at different densities and therefore, the PV-diagram shows the dominating velocity structure in different regions. This is of course no different from the single-dish spectra (Fig. 6), except that the effect is much clearer in PV-space.

\section{Results}

\subsection{Single-dish simulations}

In Fig. 9 we present the results of our model fit to the spectra from the hydrodynamical simulation using a single spectrum obtained towards the center of the source in a resolution of $15^{\prime \prime}$. This figure shows the best fit mass (left) and $\alpha$ (right) parameters for the three chosen transitions separately, and for a joint fit to all three lines simultaneously. First of all we notice that after about 1.0 free-fall time, $\mathrm{HCO}^{+}$and $\mathrm{CS}$ fail to converge, that is, the model line profile carries no resemblance to the line calculated from the hydrodynamical snapshot. However, in the case of the mass parameter, for the snapshots earlier the 1.0 free-fall time as well as for the entire sequence of CO spectra, we actually get rather accurate parameter values, although we do consistently underestimate the mass for $t<1.0 t_{\mathrm{ff}}$. The error bars or robustness of the fit becomes significantly worse towards the end of the sequence for the mass determination using $\mathrm{CO}$, but the actual best fit value is close to the correct value. The situation is more complicated if we consider the $\alpha$ parameter. Here the CS does not show any consistency at any point throughout the sequence. $\mathrm{CO}$ and $\mathrm{HCO}^{+}$are considerably better, but the best overall fit is provided by the case where all three lines are fit at the same time.

Generally we see that fitting all three lines at the same time does not give a much better fit than the best single transition provides, in this case the $\mathrm{CO}$ line. The fact that $\mathrm{CO}$ seems to be the best choice in order to accurately describe the velocity field using single-dish observations is somewhat contradictory to the result of the correlation calculations in Sect. 3.1, where the CO profile was seen to be the least correlated with the velocity field. According to those numbers, $\mathrm{HCO}^{+}$and $\mathrm{CS}$ should be able to give much more accurate results the $\mathrm{CO}$.

The problem is that when using tracers that probe much deeper into the envelope we start to probe into the regions where our simplified spherical model no longer gives an accurate description. Because we use an average density profile in order to reproduce the total cloud mass, we automatically get lower column densities in the disk region, and this means that lines can get optically thick in the hydrodynamical simulation but not in our parameterized model. This is the reason why the $\mathrm{CS}$ and $\mathrm{HCO}^{+}$ lines are no longer reproducible at $t>1.0 t_{\mathrm{ff}}$. Furthermore, in the early snapshots, CS is optically thin and the line has thus little structure, which makes it difficult to accurately fix $\alpha$.

Interestingly, it seems that a low density tracer such as $\mathrm{CO}$ gives the most accurate description of the velocity field even though the $\mathrm{CO}$ line never actually shows any rotational characteristics (e.g., compare the CO profile at $t=2.0 t_{\mathrm{ff}}$ in Fig. 6 to the lower left panel of Fig. 5), mainly because CO gets optically thick at radii larger than the outer disk radius. Another advantage of using $\mathrm{CO}$ as tracer is that it is less affected by the depletion that occurs in the high density (disk) regions because it gets 

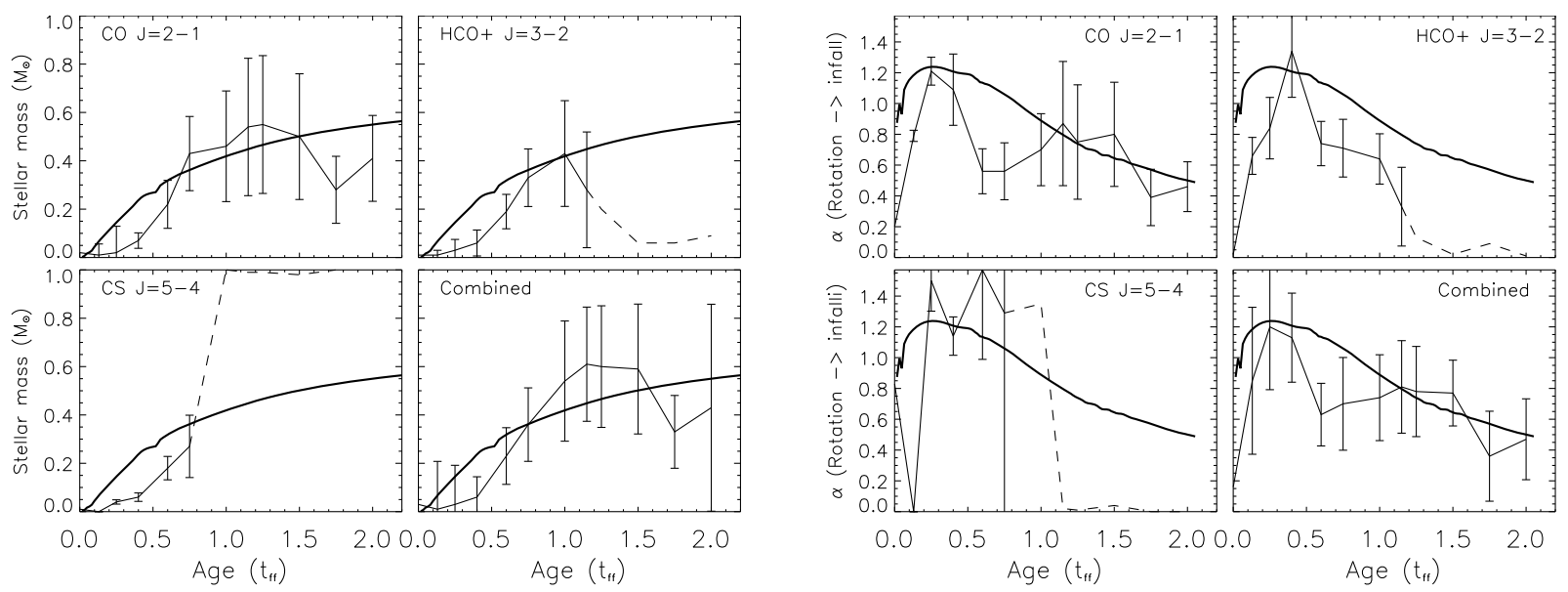

Fig. 9. The result of low-resolution model fits to spectra based on the hydrodynamical simulation. The four left panels show the best fit value for the central mass based on $\mathrm{CO}, \mathrm{HCO}^{+}$, and CS spectra as well as a fit using all three lines simultaneously. The thick solid line is the values from the simulation and the thin lines connect the best fit values. The error bars are the width of the $\chi^{2}$ distributions as discussed in Sect. 2.3. Dashed lines denotes unconverged models, i.e., cases where the line profile cannot be reproduced by our model. The four panels to the right are similar, but for the velocity angle $\alpha$ in radians as defined by Eq. (4).

optically thick further out. High density tracers such as $\mathrm{HCO}^{+}$ and especially CS probe into the region where freeze-out is significant which makes the whole process considerably more complicated. On the other hand, because $\mathrm{CO}$ is excited at low densities and temperatures, the spectrum has a much higher chance of being polluted by emission from ambient material. This can be hard to identify and if present must be accounted for in the modeling. Also, although $\mathrm{CO}$ is the best candidate of the three tracers shown here, the correlation between the derived velocity field and the real velocity field is actually not very high, with most of the points lying between 0.6 and $0.8\left(\approx 45^{\circ}\right)$.

\subsection{Interferometric simulations}

We now turn to the high-resolution situation, where we use the PV-diagram to constrain the velocity field parameters. Because the PV-diagram is a two-dimensional intensity distribution, comparing model to data is not as straight-forward as in the case of using a single spectrum, because there are several ways to evaluate a fit in PV-space.

The obvious method is to evaluate the fit by comparing the model PV-diagram to the data, pixel by pixel. While this works reasonably well when using synthetic, noiseless data which are perfectly aligned with the coordinate axes, it may prove very difficult and unreliable using real data, where the position angle may not be perfectly well determined. Also, if the model is not describing the source geometry very well, especially on small scales, a pixel by pixel comparison can easily result in a poor fit, although the velocity model is actually quite accurate. Nevertheless, we have tried using this method, just to see how well it works, when noise is not present.

Another way to obtain a fit is to determine the position of the two maxima of the intensity distribution (the blue peak and the red peak) and then use the straight line between these two points as a measure. This line is characterized by three numbers: its length, slope, and orientation in PV-space. This method is better when using real data because it works even if the data is quite noisy. Unfortunately, the minimum in $\chi^{2}$-space is less well-defined (the fitness landscape is more shallow) using this method, because of the degeneracies occurring when using just a linear gradient to evaluate the fit and therefore, in most cases, the error bars are quite large.

A third method that we have tried to use is the asymmetry in the PV-diagram as a constraint. We do this by summing up the emission in each of the four quadrants and then evaluate the fit by comparing the ratio of emission in quadrant 1 and 4 to the emission in quadrant 2 and 3 as well as the ratio of emission in 1 and 2 to 3 and 4 . In other words, we measure the left/right and top/bottom asymmetry in the PV-diagram. This method has the disadvantage that it results in a diagonal ridge in $\chi^{2}$-space, and thus a further constraint is needed. To solve this degeneracy, we use a single-dish line. However, using the entire singledish line profile together with the PV-diagram results again in a shallow fitness landscape which we are not interested in. On the other hand, we only need to constrain one parameter with the single-dish line to resolve the degeneracy, and therefore we mask the center part of the line and only fit the line wings. We mask out all channels which lie within the FWHM of the line and use the remainder to calculate a $\chi^{2}$ value which is then added to the $\chi^{2}$ value we get from the PV-diagram.

The result of these three methods are shown in Fig. 10. The three thin lines correspond to the three methods described above, with the pixel by pixel method plotted as the full line, the linear gradient as the dashed line and the emission asymmetry together with the single-dish line wings as the dotted line. The three methods more or less agree on the results, except for the mass parameter using the pixel by pixel comparison, which diverges after about 0.5 free-fall times. The main difference between the three methods is the size of the error bars (not shown in Fig. 10), which vary quite dramatically, not only from one method to the other, but also from snapshot to snapshot with the same method. Generally, the third method described above gives the smallest errors, except for a few of the earlier snapshots where the linear gradient has a better defined minimum in $\chi^{2}$-space.

Because each of our methods comes with an estimate of how well defined the fit is, for each snapshot we can select the method that is most discriminating. This is shown in Fig. 11, together with the corresponding error bars. The resulting curve describes the parameters of the hydrodynamical simulation far better than the case of the single-dish result and also better than any of the three methods individually shown in Fig. 10. The error bars are also very small in almost all of the snapshots which means that 

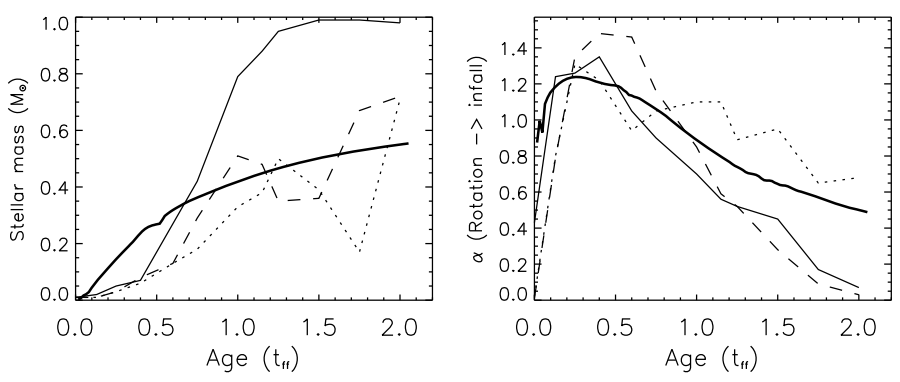

Fig. 10. Similar to Fig. 9, but for $\mathrm{HCO}^{+} 3-2$ in a resolution of $1^{\prime \prime}$, using the PV-diagram to evaluate the $\chi^{2}$. The three thin line styles correspond to three different method to evaluate the $\chi^{2}$. The full line corresponds to fitting the PV-diagram pixel by pixel, the dashed line is the fit obtained from a linear gradient in PV-space, and the dotted line is the combination of using the PV-diagram and a single-dish line.
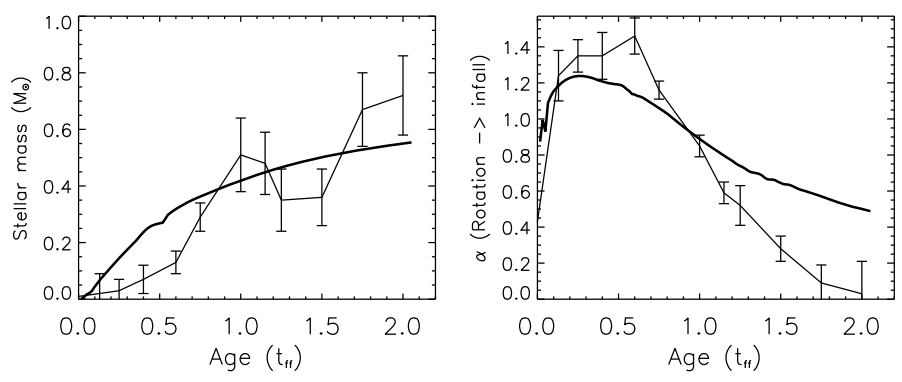

Fig. 11. High-resolution model fit, similar to that of Fig. 10. Each point shown here together with its associated error bar has been picked among the three different solutions shown in Fig. 10 using the smallest error bar as selection criterion.

the fits are very robust or in other words, we can always find a method that gives us acceptable small error bars. The main deviation in Fig. 11 is the $\alpha$-parameter in the later part of the collapse. Here, our model overestimates the amount of rotation, near the end by a factor 10 . However, the graph has a very smooth behavior compared to the best result of the single-dish lines, where the solution fluctuates around the correct values. Also, the error bars are very small, compared to those of the single-dish result, and this means that it should be possible to determine relative ages in a sample of objects using the same approach as the one we used to obtain the result in Fig. 11, even though the actual absolute value derived for $\alpha$ is incorrect.

\section{Discussion}

The above analysis has been made using synthetic data which in many respects are unrealistic in the sense that they are noiseless and uncontaminated by fore- or background material. Furthermore we have disregarded the chemistry, using a constant abundance distribution instead. In the following we will discuss the effect on our result that using real data will have.

As mentioned in Sect. 2, freeze-out chemistry is important in YSOs (Aikawa \& Herbst 2001; Bacmann et al. 2002) and this is likely what causes the most concern with respect to our results. Freeze-out occurs in regions of high density and low temperature, a condition which is present in the outer parts of the disk and inner parts of the envelope. As the molecules freeze out onto grain surfaces, they no longer contribute to the radiation field of the rotational transitions, and if the gas in the entire disk is frozen out we do not see any rotational motion at all. There is no doubt that YSOs in general are affected by some freeze-out, but the question is to what extent this freeze-out affects the spectra.
In a subsequent paper (Brinch et al. 2008) a realistic freezeout model has been added to the hydrodynamical simulation presented in this paper, in order to get time-resolved abundance profiles. In that paper only the CO molecule is considered, but the result may be generalized to include at least also $\mathrm{HCO}^{+}$because the chemistry of this molecule follows that of CO closely. The main result of that paper is that the optically thick spectra are not much affected throughout the simulation. Freeze-out occurs mainly beyond the $\tau=3$ isosurface of ${ }^{12} \mathrm{CO}$. This is interesting since the $\tau=3$ surface is located at the same physical radius no matter whether there is freeze-out within this radius or not. This means that in our models too, we never actually probe the disk regions directly, and thus we conclude that when we seem to measure rotation, we are actually rather measuring lack of infall.

On the other hand, optically thin lines are quite strongly affected by the freeze-out zones, because these lines probe the full column density. In Sect. 2 we described how we use an optically thin isotopologue to determine the average abundance, and therefore a change in an optically thin line due to freeze-out will cause a wrong abundance determination which will then propagate to the optically thick lines that are used to obtain the velocity field. If freeze-out is present in the data, we can either still try to fit it with a constant abundance profile or we can add a simple parameterized freeze-out model, following the approach of Jørgensen et al. (2005b). In the former situation we derive an average abundance which is about a factor of two lower than the correct value for the undepleted zones, whereas if we allow for freeze-out in our model as well, we derive a gas-phase abundance which is about $20 \%$ lower than the correct abundance. This should be compared with the situation described in Sect. 2 where we had no freeze-out and we were able to derive the abundance to within $1 \%$. However, underestimating the abundance by a factor of 2 does not make a big difference for the continued analysis and therefore it should be safe to use a flat abundance distribution in the model, even though the object in question is known to have depletion zones. It is of course also always possible to take a non-constant abundance profile into consideration when modeling sources for which the chemistry is already known (e.g., as in the case of NGC 1333-IRAS2, Jørgensen et al. 2005a).

Another source of confusion is contamination from foreground material (Boogert et al. 2002), ambient cloud material or outflows (Bally \& Lada 1983). Because larger scale mapping can identify the former of the two, only the outflows are a matter of real concern. Outflow emission shows up as a broadening and enhancement of the line wings and, if present, is typically seen in tracers such as $\mathrm{CO}$ and $\mathrm{HCO}^{+}$. Only single-dish lines are really affected by these effects, because interferometers typically resolve out large scale structure, such as ambient cloud material, and outflow regions are easily identifiable and can be masked when making the PV-diagram. Foreground material is less of a concern if $\mathrm{HCO}^{+}$is used, because this molecule is only excited at densities higher than what is typical for diffuse interstellar foreground layers. For single-dish lines, ambient material may shown up as excess emission near the line center which makes it impossible to use the line profile to trace the velocity field. If, however, only the line wings are used, ambient or foreground material should not be a problem. We are thus left with the outflow contamination of the single-dish lines, which mainly affect the line wings.

If a source is known to drive a strong outflow, or if inconsistent results are obtained when fitting the model with and without the use of a single-dish line, it is necessary to either use a different single-dish line that is less affected by the outflow or simply 
to avoid using single-dish lines for that particular source. This may result in a less strict result, but there is no way to reliably remove the contribution of an outflow from a spectral line unless the central mass and to some extent also the velocity field (and thus the shape of the line wing) are already known.

Finally, real data are noisy and this may cause confusion when fitting the single-dish lines. As was mentioned above, using a linear gradient or comparing the total emission in the four quadrants of the PV-diagram is little affected by the presence of noise. However, for the single-dish data it is important to use strong lines ( $\mathrm{CO}$ and $\mathrm{HCO}^{+}$are almost always strong) and integrate long enough so that the line wings have good signal-tonoise ratio. If this is not possible, it is again simply a matter of using the PV-diagram without the constrain of a single-dish line, at the price of a less certain result.

\section{Conclusion}

We have explored the possibility of placing Young Stellar Objects in an evolutionary ordering based on their kinematical configurations by fitting a simple parameterized model to submillimeter observations. We have tested the feasibility of this by fitting the simple velocity model to a time series of synthetic observations with known velocity fields, generated by a hydrodynamical simulation. We find that the model reproduces the synthetic spectra reasonably well and that the best fit parameters which describe the velocity field are in agreement with the velocity field in the hydrodynamical simulation. We therefore conclude that it is possible to extract reliable information on astronomical objects if we replace the synthetic spectra with real observations.

We find that it is difficult, though not impossible, using single-dish data alone, due to the shallowness of the $\chi^{2}$-space, but feasible if interferometric data are used, especially when combined with one or more single-dish lines. Using the latter option, we find that the central dynamical mass can be determined to within $20 \%$. However, we have not been able to establish a single "best way" to obtain the parameters throughout the collapse. We find that the best result is obtained by trying several different methods to constrain the fits, and then pick the result of the one which shows the most peaked $\chi^{2}$-space. That said, most of our best constrained points were obtained using a combination of low-resolution and high-resolution and this is the preferred starting point.

We further conclude that the molecular species used are not crucial for the result, as long as the line wings are clearly defined and uncontaminated in the case of the single-dish lines, and also that the transition is optically thick. For the high-resolution data, signal-to-noise is the main concern, so strong lines are preferred $\left(\mathrm{CO}, \mathrm{HCO}^{+}\right.$, etc.).

Due to the uncertainty of the absolute time scale of the collapse calculated by our simple hydrodynamical simulation, we cannot yet establish an absolute age of a given object, but relative ages among a sample of objects can be obtained, provided that the velocity field of a collapsing cloud evolves similar to that of the simulation. Indeed, if an age calibration could be made, for example using the chemical properties of one or more objects, this could prove a powerful method to place a large number of young stars in an evolutionary sequence, because of the straightforward observations needed to perform the analysis. The hydrodynamical simulation presented in this paper is not required in order to analyze real objects using our method. However, with an improved and more realistic hydrodynamical scheme, it might be possible to calibrate the evolution of the velocity field so that an absolute time scale of star formation can be established.

Acknowledgements. C.B. is partially supported by the European Commission through the FP6 - Marie Curie Early Stage Researcher Training programme. The research of M.R.H. is supported through a VIDI grant from the Netherlands Organization for Scientific Research.

\section{References}

Aikawa, Y., \& Herbst, E. 2001, A\&A, 371, 1107

Bacmann, A., Lefloch, B., Ceccarelli, C., et al. 2002, A\&A, 389, L6 Bally, J., \& Lada, C. J. 1983, ApJ, 265, 824

Basu, S. 1998, ApJ, 509, 229

Belloche, A., André, P., Despois, D., \& Blinder, S. 2002, A\&A, 393, 927

Bodenheimer, P., Yorke, H. W., Rozyczka, M., \& Tohline, J. E. 1990, ApJ, 355, 651

Boogert, A. C. A., Hogerheijde, M. R., Ceccarelli, C., et al. 2002, ApJ, 570, 708 Brinch, C., Crapsi, A., Hogerheijde, M. R., \& Jørgensen, J. K. 2007, A\&A, 461, 1037

Brinch, C., van Weeren, R. J., \& Hogerheijde, M. R. 2008, A\&A, 489, 617

Calvet, N., \& Hartmann, L. 1992, ApJ, 386, 239

Cassen, P., \& Moosman, A. 1981, Icarus, 48, 353

Charbonneau, P. 1995, ApJS, 101, 309

Evans, II, N. J. 1999, ARA\&A, 37, 311

Gregersen, E. M., \& Evans, N. J. 2000, ApJ, 538, 260

Hogerheijde, M. R. 2001, ApJ, 553, 618

Hogerheijde, M. R., \& van der Tak, F. F. S. 2000, A\&A, 362, 697

Jørgensen, J. K., Schöier, F. L., \& van Dishoeck, E. F. 2004, A\&A, 416, 603

Jørgensen, J. K., Bourke, T. L., Myers, P. C., et al. 2005a, ApJ, 632, 973

Jørgensen, J. K., Schöier, F. L., \& van Dishoeck, E. F. 2005b, A\&A, 435, 177

Keto, E. R. 1990, ApJ, 355, 190

Lada, C. J., \& Wilking, B. A. 1984, ApJ, 287, 610

Lee, J.-E., Bergin, E. A., \& Evans, II, N. J. 2004, ApJ, 617, 360

Lommen, D., Jørgensen, J. K., van Dishoeck, E. F., \& Crapsi, A. 2008, A\&A, 481,141

Mardones, D., Myers, P. C., Tafalla, M., et al. 1997, ApJ, 489, 719

Myers, P. C., Mardones, D., Tafalla, M., Williams, J. P., \& Wilner, D. J. 1996, ApJ, 465, L133

Ohashi, N., Hayashi, M., Ho, P. T. P., \& Momose, M. 1997, ApJ, 475, 211

Różyczka, M. 1985, A\&A, 143, 59

Saito, M., Kawabe, R., Kitamura, Y., \& Sunada, K. 1996, ApJ, 473, 464

Schöier, F. L., van der Tak, F. F. S., van Dishoeck, E. F., \& Black, J. H. 2005,

A\&A, 432, 369

Shakura, N. I., \& Sunyaev, R. A. 1973, A\&A, 24, 337

Shu, F. H. 1977, ApJ, 214, 488

Terebey, S., Shu, F. H., \& Cassen, P. 1984, ApJ, 286, 529

Ulrich, R. K. 1976, ApJ, 210, 377

van Langevelde, H. J., van Dishoeck, E. F., \& Blake, G. A. 1994, ApJ, 425, L45

Yorke, H. W., \& Bodenheimer, P. 1999, ApJ, 525, 330

Yorke, H. W., Bodenheimer, P., \& Laughlin, G. 1993, ApJ, 411, 274

Yorke, H. W., Bodenheimer, P., \& Laughlin, G. 1995, ApJ, 443, 199 